\title{
The high SNR rate in the Galactic Center: origin of the cosmic rays excess?
}

\author{
L. Jouvin, A. Lemière, and R. Terrier \\ APC, UMR 7164, CNRS, Université Paris VII, CEA, Observatoire de Paris, Paris \\ email: lea.jouvin@apc.in2p3.fr
}

\begin{abstract}
The center of our Galaxy hosts a Super-Massive Black Hole (SMBH) of about $4 \times 10^{6}$ $\mathrm{M}_{\odot}$. Since it has been argued that the SMBH might accelerate particles up to very high energies, its current and past activity could contribute to the population of Galactic cosmic-rays (CRs). Additionally, the condition in the Galactic Center (GC) are often compared with the one of a starburst system. The high supernovae (SN) rate associated with the strong massive star formation in the region must create a sustained CR injection in the GC via the shocks produced at the time of their explosion.

The presence of an excess of very high energy (VHE) cosmic rays in the inner $100 \mathrm{pc}$ of the Galaxy in close correlation with the massive gas complex known as the central molecular zone (CMZ) has been revealed in 2006 by the H.E.S.S. collaboration. Recently, by analysing 10 years of H.E.S.S. data, the H.E.S.S. collaboration confirmed the presence of this extended VHE diffuse emission and deduced a CR density peaked toward the GC. The origin of the CR over-abundance in the GC still remains mysterious: Is it due to a single accelerator at the center or to multiple accelerators filling the region?

In order to investigate the presence of these multiple CR accelerators, and in particular the impact of their spatial distribution on the VHE emission morphology, we build a 3D model of $\mathrm{CR}$ injection and diffusive propagation with a realistic 3D gas distribution. We discuss the CR injection in the region by a spectral and morphological comparison with H.E.S.S. data.

We show that a peaked $\gamma$-ray profile towards the GC center is obtained using a realistic SN spatial distribution taking into account the central massive star clusters. The contribution of theses sources cannot be neglected in particular at high longitudes. In order to fit the very central excess observed with H.E.S.S., another central VHE component is probably necessary.
\end{abstract}

\section{Introduction}

Recently, Abramowski et al. (2016) confirmed the presence of an extended VHE emission (100 GeV-100 TeV) in the Galactic Center (GC) detected by the High Energy Stereoscopic System (H.E.S.S.). This diffuse emission is correlated with the matter distribution in this region called the Central Molecular Zone (CMZ) which contains around $\sim 10 \%$ of the total molecular mass of the Galaxy in a rather complex setup of dense molecular clouds (density $\approx 10^{4} \mathrm{~cm}^{-3}$ ). The close correlation of this emission with the target material suggests that the dominant component of the ridge is due to the interaction of relativistic cosmic rays (CRs) with protons in the ambient medium. The high amount of CRs deduced from this emission is between 3 and 9 times higher that of the flux measured on Earth with a harder spectrum of $\Gamma \sim 2.3$.

The center of our Galaxy hosts a Super-Massive Black Hole (SMBH), SgrA*, of about $4 \times 10^{6} \mathrm{M}_{\odot}$ (Ghez et al. 2008). This SMBH is extremely inactive and is fed by the stellar winds in this region. With an actual bolometric luminosity of $10^{36} \mathrm{erg}$, much of the accretion power in the bondi region, $10^{39} \mathrm{erg} \mathrm{s}^{-1}$ (Wang et al. 2013), is probably used to accelerate $\mathrm{CR}$ up to $\mathrm{PeV}$ energies in the surrounding powerful outflows (Wang et al. 2013; Liu et al. 2006). 
The condition in the GC are often compared with the one of a starburst system. Many Supernova Remnants (SNR) are visible through their radio and X-ray thermal emission (Ponti et al. 2015). The SN rate determined from stellar composition by Crocker et al. (2011) goes from 0.02 to 0.08 SN per century. Knowing that the kinetic energy released from a SN explosion is $10^{51} \mathrm{erg}$ and assuming a recurrence time of 2500 year, the injection power in the GC from these sources is around $10^{40} \mathrm{erg} \mathrm{s}^{-1}$. The $\gamma$-ray luminosity observed by H.E.S.S. is several order of magnitude lower than this input power (Crocker et al. 2011). Therefore, any model of $\gamma$-ray emission in the region has to take into account the propagation and the escape of CR injected by these multiple accelerators.

This work aims to study the impact of SNRs in the VHE emission observed in the inner $200 \mathrm{pc}$ of the Galaxy and in particular the impact of their spatial distribution on the CR density. The presence of three young clusters at the center, the Quintuplet, 3-5 Myrs old (Najarro et al. 2004, the Arches, 2-3 Myrs old (Figer et al. 1999) and the Central disk surrounding SgrA ${ }^{\star}$, 4-6 Myrs old (Liu et al. 2013) indicates that even if the real SN spatial distribution is unknown, it is clearly non uniform and concentrated to the center. It could therefore also create a CR density peaked toward the GC.

We want to emphasize that any scenario of CR injection at the center (Macias et al. 2014; Abramowski et al. 2016) cannot be studied separately from these SNRs contributions. Given typical diffusion coefficient values in the interstellar medium (Aharonian 2004), diffusive escape is dominant over advective escape. To take into account the impact of the actual matter and SNR distribution, we build a 3D model of CR and gas distribution. We discuss how the spatial distribution of random impulsive injections throughout the CMZ modelling the SNR contributes to the CR excess seen by H.E.S.S. compare to a stationary source at the center modelling $\operatorname{Sgr}^{\star}$.

\section{3D model: setup and physical parameters}

One of the key point for studying the CR distribution is the temporal and the spatial distribution of the SNe filling the GC. We generate impulsive sources distributed according to a Poisson law of recurrence time $\tau=2500$ years. We keep only the SNe with an age $>1$ kyr since no younger SN has been observed in the GC and $<100$ kyrs since for larger times the CR density becomes negligible. The SNR spatial distribution is modelised with two components: a uniform one for the evidence of the high isolated massive stars since around $1 / 3$ of the massive stars detected in the GC are located outside of the three massive starburst clusters (Mauerhan et al. 2010) with a concentration of the SNe in the clusters, the Quintuplet and the Central Cluster. The Arches cluster has probably not experienced any supernova. We therefore do not include a concentration of SNe at this position. The SN rate in both clusters is determined from physical observations (Jouvin et al. 2016). We also investigate the scenario of a single stationary accelerator at the center to model SgrA* since Abramowski et al. (2016) have shown that the CR density profile deduced from H.E.S.S. data is compatible with a $1 / \mathrm{r}$ profile as the one expected from a point stationary source at the GC.

We model the CR propagation in the GC assuming an isotropic diffusion (Jouvin et al. 2016). We consider a power-law diffusion coefficient: $\mathrm{D}=D_{o}(E / 10 \mathrm{TeV})^{d}$ and we assume Kolmogorov turbulences $(\mathrm{d}=0.3)$. The structured magnetic field in the $\mathrm{GC}$ indicates the possibility of high diffusion coefficient values. The value assumed in this study is $D_{o}=2 \times$ $10^{29} \mathrm{~cm}^{2} \mathrm{~s}^{-1}$ close to some interstellar medium value (Aharonian 2004). The convection is thus negligible for these high energy protons. The 3D Green function, obtained assuming a CR density equal to zero at $r=+\infty$, gives the solution for an impulsive accelerator as the SNe. Integrating the previous Green function gives the solution for a continuous 
Table 1. Physical parameters used for our 3D model of the CR injection and propagation

\begin{tabular}{|l|l|}
\hline Model Parameters & Values \\
\hline Proton spectral index & 2 \\
$E_{\text {max of the injected proton }}$ & $1 \mathrm{PeV}$ \\
spectrum & $500 \mathrm{p} c \times 500 \mathrm{p} c \times 50 \mathrm{p} c$ \\
Box size & $4 \times 10^{7} \mathrm{M} \odot$ \\
Total gas mass & $2 \times 10^{29} \mathrm{~cm}^{2} \mathrm{~s}^{-1}$ \\
$D_{0}(10 \mathrm{TeV})$ & 0.3 \\
Spectral index of the diffusion & \\
coefficient (d) & $10^{51} \mathrm{erg}$ \\
$E_{S N}$ & $2500 \mathrm{yrs}$ \\
SN recurrence time & \\
\hline
\end{tabular}

source. We assume a power-law CR injection with a spectral index $a$ equal to 2 since this value is reproduced by the first order Fermi acceleration for strong shocks. Time dependent CR escape from the SNR shock is not taken into account. SNe are modelled by impulsive CR injections emitted at the same time whatever their energy.

In order to model the 3D $\gamma$-rays distribution produced by the interaction of the CRs with the matter in the GC, one has to build a coherent $3 \mathrm{D}$ matter distribution. Since our knowledge of the true gas kinematics in the GC is very limited, we will base our gas distribution (Jouvin et al. 2016) on the work of Sawada et al. (2004) who derived a face-on-map of the molecular gas without any kinematics assumption, relying only on two lines surveys (comparison between the $\mathrm{CO} 2.6-\mathrm{mm}$ emission with the $\mathrm{OH} 18-\mathrm{cm}$ absorption). The table 1 contains the different physical parameters used for our model and more details are given in Jouvin et al. (2016). In the following, we place constrains on the CR injection and propagation through a comparison with the existing H.E.S.S. data in the region.

\section{Result}

Using reasonable parameters on the SN rate and the diffusion, both our models reproduce the total spectrum observed with H.E.S.S. in the region (Abramowski et al. (2016)) as observed on the figure 1.a. Considering only the SNRs in the GC, we have to consider a quite low acceleration efficiency of $2 \%$ percent of the kinetic energy released from a SN explosion in order to not over produce the total $\gamma$-rays flux. The power needed for CR acceleration for the stationary source is compatible with the one found in Abramowski et al. 2016 around $10^{38} \mathrm{erg} \mathrm{s}^{-1}$ making this central source a good candidate to the VHE CR acceleration in the GC. .

We then directly compare the CR density profile of our models with the one obtained from H.E.S.S. data. The figure 1.b presents the average CR enhancement within seven circular regions (Abramowski et al. 2016) at different distance from SgrA* compared with the local CR energy density measured in the Solar neighbourhood at those energies. In order to compare our models with these results, we determine the average energy density of CR above $10 \mathrm{TeV}$ along the LOS by weighting the CR density by our matter distribution (Jouvin et al. 2016).

Taking into account a realistic SNe and matter spatial distributions provides a peaked $\gamma$-ray profile and a pronounced CR gradient as seen in Figure 1.b. The SNe have a major contribution to the VHE emission in particular to larger longitudes. The observed CR profile seems more peaked than the profile predicted by SNe alone. A second component is probably required to explain the very central excess. As proposed by Abramowski et al. (2016), the GC stationary source alone can well explain the CR profile, but this requires 


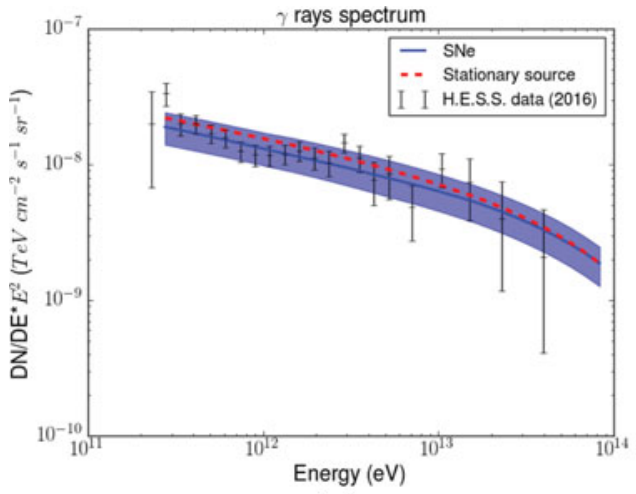

(a)

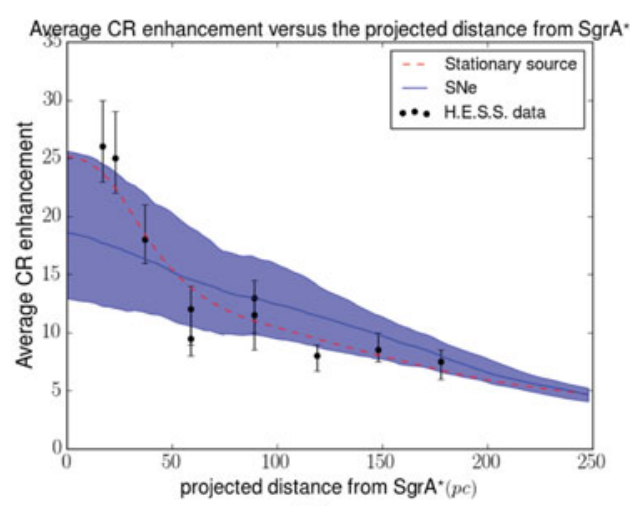

(b)

Figure 1. (a) Median of the spectrum extracted from an annulus centred at $\operatorname{SgrA}^{\star}$ with inner and outer radii of $0.15^{\circ}$ and $0.45^{\circ}$ of the realisations of the $100 \mathrm{SN}$ temporal and spatial distributions (solid line) as well as the dispersion around the median and the spectrum for a stationary source at the GC (dash line). (b) Average CR enhancement within seven circular regions of radius 0.1 degrees distributed along the Galactic Plane (Abramowski et al. 2016) compared with the local CR energy density measured in the Solar neighbourhood at those energies which is $w_{0}(>10 \mathrm{TeV}) \approx 10^{-3} \mathrm{eV} / \mathrm{cm} 3$. Average CR enhancement for a stationary source situated at the GC (dash line) and for the SNe modelling taking into account the two massive cluster for the spatial distribution (solid line). These profiles are the mean of the profiles for galactic latitudes b: $|b|<0.1^{\circ}$. The black points in (a) and (b) are the HESS data from Abramowski et al. (2016).

negligible contribution from SNe in order to not overproduce the total $\gamma$-ray flux and CR density estimated with the H.E.S.S. data. This is at odds with the already low acceleration efficiency required to not over-produce the total $\gamma$-ray spectrum. However, regarding the very hot medium the shock following the explosion could be weakly supersonic. Therefore a scenario of ultra-low efficient $\mathrm{SN}$ is not to exclude.

\section{References}

A. Abramowski, et al., 2016, Nature, 531, 476

F. Aharonian, 2004

G. Ponti, et al., MNRAS, 453, 172

F. Aharonian, et al. 2006, Nature, 439, 695-698

A. M. Ghez, et al. 2008, ApJ, 689, 1044-1062

Q. D. Wang, et al. 2013, Science, 341, 981-983

S. Liu, et al. 2006, ApJ, 647, 1099-1105

R. M. Crocker, et al. 2011, MNRAS, 413, 763-788

O. Macias, et al. 2014, Arxiv e-prints

J. C. M.auerhan, et al. 2010, ApJ, 725, 188-199

F. N.ajarro, et al. 2004, ApJ, 611, L105-L108

H. B. Liu, et al. 2013, ApJ, 770, 44

Figer, et al. ApJ, 525, 750

T. Sawada, et al. 2004, MNRAS, 349, 1167-1178

L. Jouvin, et al. 2016, MNRAS, submitted 